\title{
Development of a novel enzyme-targeting radiosensitizer (KORTUC) containing hydrogen peroxide for intratumoral injection for patients with low linear energy transfer-radioresistant neoplasms
}

\author{
SHIHO TOKUHIRO, YASUHIRO OGAWA, KAZUHIRO TSUZUKI, RYO AKIMA, \\ HIRONOBU UE, SHINJI KARIYA and AKIHITO NISHIOKA

\begin{abstract}
Department of Diagnostic Radiology and Radiation Oncology, Medical School, Kochi University, Nankoku 783-8505, Japan
\end{abstract}

Received June 25, 2010; Accepted September 7, 2010

DOI: $10.3892 / \mathrm{ol} .2010 .184$

\begin{abstract}
The therapeutic effect of radiotherapy using linear accelerators for relatively large tumors of more than several centimeters in diameter is reduced to one third due to a large number of hypoxic tumor cells and a significant amount of anti-oxidative enzymes including peroxidase/catalase. The most effective method by which to inject hydrogen peroxide into tumor tissue was examined. This proved difficult as $3 \% \mathrm{w} / \mathrm{v}$ hydrogen peroxide solution (Oxydol) is an antiseptic agent for skin lesions. Thus, injection into an affected lesion may result in hydrogen peroxide soaking into a body cavity, possibly causing an intra-arterial oxygen embolism. This study aimed to identify the most effective combination of drugs containing hydrogen peroxide in order to relieve local pain at the injection site and preserve high intratumoral oxygen concentration. Hyaluronate-hydrogen peroxide was identified as the most effective combination of drugs containing hydrogen peroxide for the preservation of high intratumoral oxygen concentration for $24 \mathrm{~h}$ following intratumoral injection with the agent. Based on the results, the clinical application of a novel enzyme-targeting radiosensitization treatment, Kochi Oxydol-Radiation Therapy for Unresectable Carcinomas (KORTUC), was initiated for malignant tumors including advanced breast cancer, soft tissue sarcoma and cervical lymph node metastasis. Moreover, we have developed KORTUC III for locally advanced hepatocellular carcinoma and KORTUC IV for locally advanced pancreas cancer (stage IVa).
\end{abstract}

Correspondence to: Professor Yasuhiro Ogawa, Department of Diagnostic Radiology and Radiation Oncology, Medical School, Kochi University, Nankoku 783-8505, Japan

E-mail: ogaway@kochi-u.ac.jp

Key words: radiosensitizer, hydrogen peroxide, Kochi OxydolRadiation Therapy for Unresectable Carcinomas, sodium hyaluronate, radiation, intratumoral injection

\section{Introduction}

Low linear energy transfer (LET)-radiation, including $\mathrm{X}$-rays and electron beams from linear accelerators, is used worldwide for clinical radiotherapy. However, the therapeutic effect of radiotherapy using linear accelerators for relatively large tumors of more than several centimeters in diameter, decreases to one third than that for smaller tumors due to the presence of numerous hypoxic tumor cells and an abundance of anti-oxidative enzymes including peroxidase and catalase (1). This reduction occurs because approximately two thirds of the therapeutic effect of linear accelerators is due to the indirect effects of X-rays and/or electrons, which are mainly caused by radical formations such as hydroxyl radicals in the cytoplasm produced by radiation degradation of the intrabody and intracellular water molecules (1). Therefore, in the absence of oxygen in the tumor tissues, the effect of the X-rays and electrons decreases to one third of standard efficacy. In these situations, the use of a low concentration of hydrogen peroxide for low LET-radioresistant neoplasms causes anti-oxidative enzymes in the tumor tissues, including peroxidase and catalase, to be inactivated and, concomitantly, oxygen is produced by the degradation of hydrogen peroxide, thereby resulting in the hypoxic tumor tissue being reoxygenated.

Strong radiosensitizing effects of hydrogen peroxide have been demonstrated in this experimental study using the low LET-radioresistant osteosarcoma cell line HS-Os-1 (2-4) and a radioresistant prostatic cancer cell line (5). On the basis of these experimental results, a novel enzyme-targeting radiosensitization treatment termed Kochi Oxydol-Radiation Therapy for Unresectable Carcinomas (KORTUC) was developed. In KORTUC I, which uses a hydrogen peroxide solution (Oxydol)-soaked gauze bolus, notable therapeutic effects of hydrogen peroxide as a radiosensitizer for superficially exposed and unresectable neoplasms were obtained, as reported in a previous study (6).

The most effective method of injecting hydrogen peroxide into tumor tissue was then examined. This proved difficult as $3 \% \mathrm{w} / \mathrm{v}$ hydrogen peroxide solution (Oxydol) is an antiseptic 


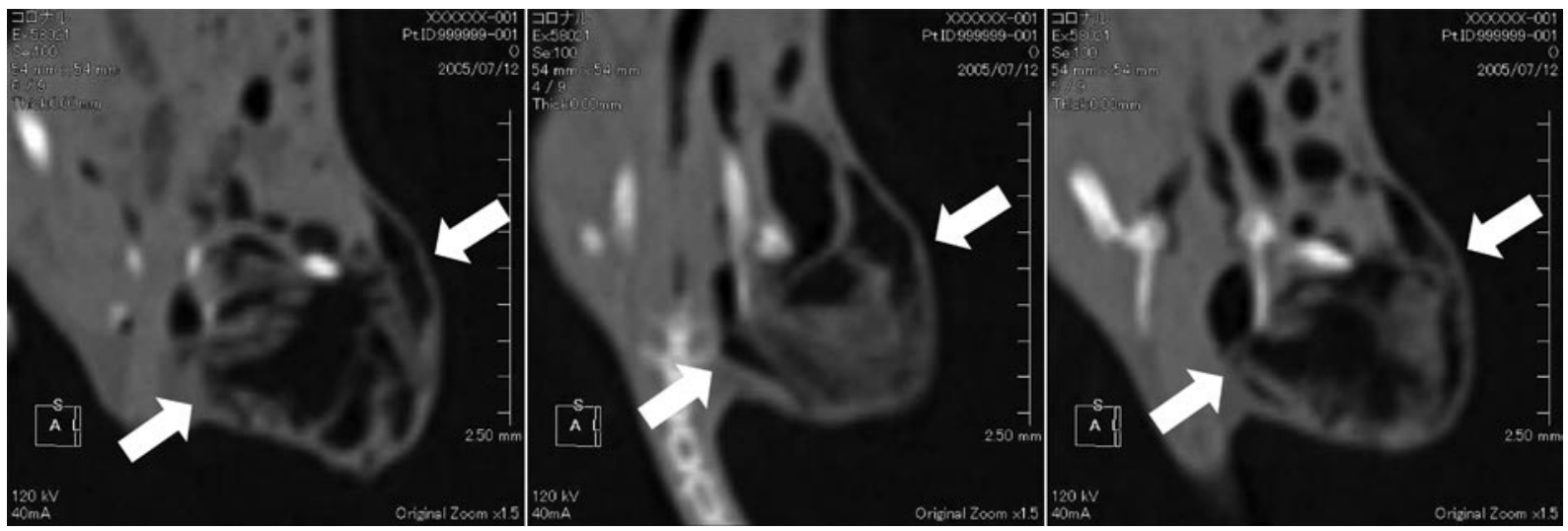

Figure 1. PBS containing $0.5 \% \mathrm{w} / \mathrm{v}$ hydrogen peroxide was injected into mouse thigh tumors. We confirmed the formation of oxygen bubble following an intratumoral injection of $1 \mathrm{ml}$ of $0.5 \% \mathrm{w} / \mathrm{v}$ hydrogen peroxide into mouse tumors using multi-row detector computerized tomography (MDCT).

agent for skin lesions, and thus injection into an affected lesion may result in hydrogen peroxide soaking into a body cavity and possibly causing an intra-arterial oxygen embolism. Regarding intratumoral hydrogen peroxide injection (7), $3-5 \mathrm{ml}$ of $3 \% \mathrm{w} / \mathrm{v}$ Oxydol was administered for meningioma with no significant side effects. Notably, the formation of oxygen bubble following an intratumoral injection of $1 \mathrm{ml}$ of $0.5 \% \mathrm{w} / \mathrm{v}$ hydrogen peroxide into mice tumors using multi-row detector computerized tomography (MDCT) was confirmed (Fig. 1).

Local pain appeared to be strong as the hydrogen peroxide was rapidly degraded by the peroxidase and catalase in the tumor tissue. Furthermore, the diffusion of hydrogen peroxide into surrounding tissues necessitates frequent injections to maintain the oxygen concentration in the tumor tissue, if hydrogen peroxide is used alone. However, if the local pain can be relieved and the oxygen concentration in the tumor can be maintained for at least $24 \mathrm{~h}$ following an intratumoral injection of hydrogen peroxide, patient stress can be largely reduced by lowering the frequency of hydrogen peroxide injections.

This study therefore aimed to identify the most effective combination of drugs containing hydrogen peroxide for relief of local pain at the injection site and to preserve high intratumoral oxygen concentration.

\section{Materials and methods}

Drug/agent combinations. To examine various drug/ agent combinations, PBS alone (control), PBS containing $0.5 \% \mathrm{w} / \mathrm{v}$ hydrogen peroxide (PBS-hydrogen peroxide), $0.8 \% \mathrm{w} / \mathrm{v}$ sodium hyaluronate containing $0.5 \% \mathrm{w} / \mathrm{v}$ hydrogen peroxide (hyaluronate-hydrogen peroxide), $0.8 \% \mathrm{w} / \mathrm{v}$ liposome containing $0.5 \% \mathrm{w} / \mathrm{v}$ hydrogen peroxide (liposome-hydrogen peroxide), $0.8 \% \mathrm{w} / \mathrm{v}$ gelatin (Gelpart) containing $0.5 \% \mathrm{w} / \mathrm{v}$ hydrogen peroxide (gelatin-hydrogen peroxide) and $0.8 \% \mathrm{w} / \mathrm{v}$ glycerol containing $0.5 \% \mathrm{w} / \mathrm{v}$ hydrogen peroxide (glycerolhydrogen peroxide) were prepared immediately prior to the injection of each agent. First, $\sim 10^{5}$ SCCVII tumor cells were inoculated into the right thigh of each $\mathrm{C} 3 \mathrm{H} / \mathrm{He}$ mouse. Once each tumor had grown to $\sim 10 \mathrm{~mm}$ in diameter, an intratumoral injection of $0.25 \mathrm{ml}$ of one of the drug combinations mentioned above was administered. The intratumoral oxygen concentration was then measured using IMP-211 apparatus (Inter Medical Co., Ltd., Japan) immediately, 1 and $24 \mathrm{~h}$ after injection of the test agents.

\section{Test agents}

ARTZ dispo. ARTZ dispo is the injection agent for sodium hyaluronate for knee joints and has been shown to improve joint function (8). This agent contains sodium hyaluronate at a concentration of $1 \% \mathrm{w} / \mathrm{v}$, and has therapeutic effects for chronic osteoarthritis of knee joints, frozen shoulder and knee pain due to rheumatoid arthritis. ARTZ dispo protects the joint tissue by covering the latter and by improving lubrication. This injection agent soaks into degenerating cartilage, thereby inhibiting degeneration of cartilage and improving its metabolism. Moreover, ARTZ dispo permeates into the synovial tissue to control inflammation and degenerative changes, and consequently relieves pain. This product provides remission of pain, an increase in daily life activities, and improvement in the range of motion in the joint.

Gelpart. Gelpart is porous gelatin composed of gelatin and the embolus medicine used for transcatheter arterial embolization therapy for hepatocellular carcinoma patients (9). The effect of a long-term embolization is evident for several days to weeks. Gelpart physically forms an embolus on the intravascular lumen of the hepatic artery that provides nourishment to the tumor through a catheter. Therefore, Gelpart induces tumor avascular necrosis.

Liposomes. Liposomes are closed endoplasmic reticula (ER) that form when phosphatide, the ER architectural component, is dissolved in water (10). Liposomes are used as a drug delivery system, i.e., technologies that deliver the amount of medicine required to the relevant part of the body at the appropriate time. The drug can be sent to a specific part of the body by enclosing the medicine in a film or in the corpuscle that forms when a lipid is distributed in water.

Glycerol. Glycerol is a concentrated glycerin and fructose injection liquid used to treat intracranial hypertension and intracranial edema when there is a reduction in brain capacity during cerebral surgery and when a decrease in the pressure of the interior of the eye is required (11). Glycerin dissolves slightly in ether and easily in water and is highly hygroscopic. Moreover, by hyperosmosis dehydration action, 


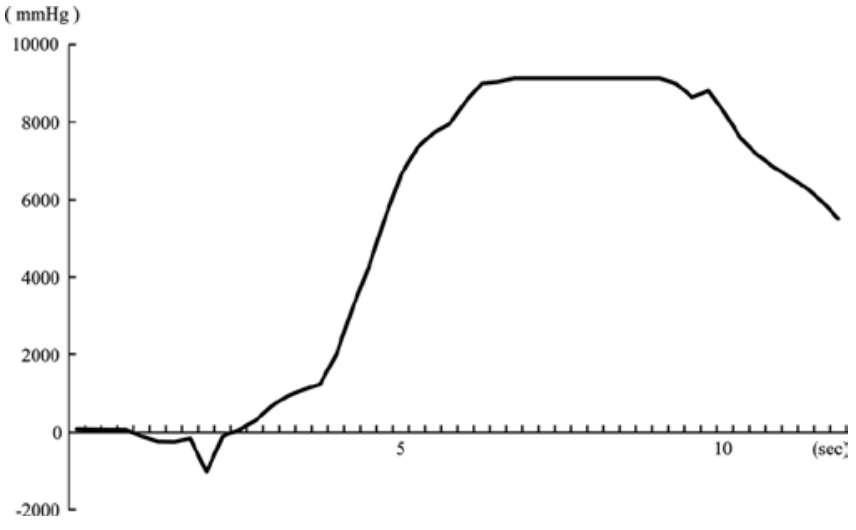

Figure 2. Oxygen concentration immediately following the intratumoral injection of PBS containing $0.5 \% \mathrm{w} / \mathrm{v}$ hydrogen peroxide. The oxygen concentration increased rapidly $\sim 4 \mathrm{sec}$ after the intratumoral injection of PBS-hydrogen peroxide.

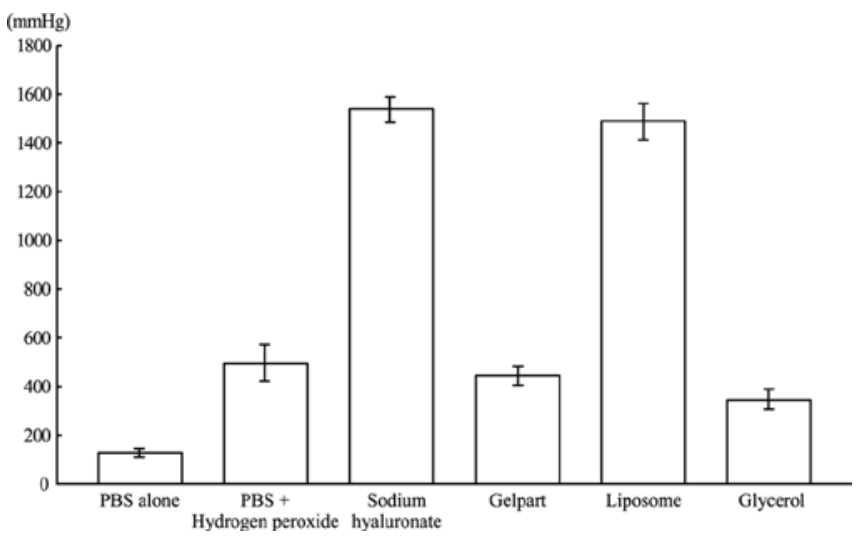

Figure 3. Oxygen concentration in the tumor tissue at $1 \mathrm{~h}$ after injection of each agent. The hyaluronate- and liposome-hydrogen peroxide-injected groups were found to preserve high intratumoral oxygen pressure.

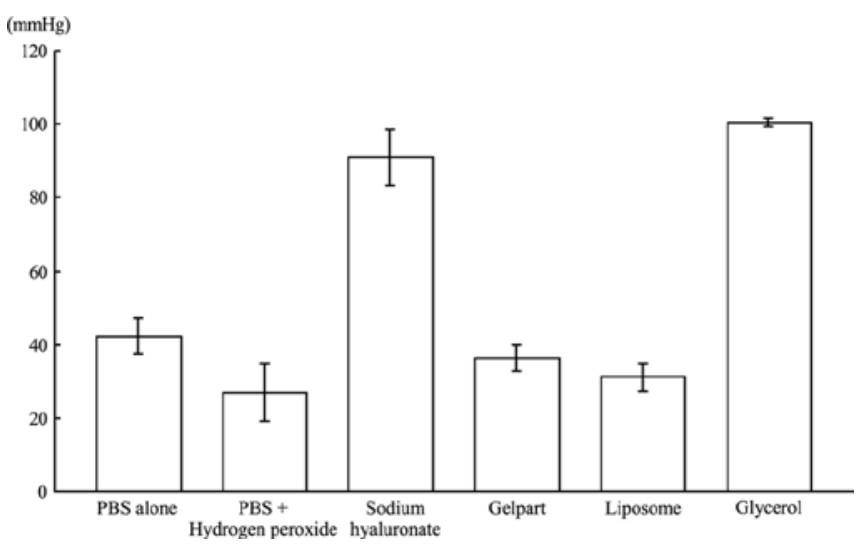

Figure 4. Oxygen concentration in the tumor tissue at $24 \mathrm{~h}$ after injection of each agent. The hyaluronate- and glycerol-hydrogen peroxide-injected groups were the only groups to show preserved high intratumoral oxygen pressure at $24 \mathrm{~h}$ after injection.

glycerin channels edema fluid to the blood vessels from brain tissues by raising the osmotic pressure of the blood when administered intravenously. Therefore, it is used widely as a therapeutic agent for acute strokes.
IMP-211. The IMP-211 oxygen concentration monitor is used to measure oxygen concentration, based on polarography. Polarography is an electrolytic analysis, in which adequate voltage is applied between the working and reference electrodes in order to conduct electrolysis. The current that flows in the electrodes is then measured, thereby obtaining a voltage-current characteristic curve. Utilizing the chemical reaction described below, the diffusion current is measured and then converted into oxygen concentration. Platinum is used as the working electrode and $\mathrm{Ag}-\mathrm{AgCl}$ is used as the reference electrode. Platinum consumes oxygen, allowing for current to flow. Based on the correlation between the consumption of oxygen and the current, the oxygen concentration can be measured. Furthermore, the electrode tip is coated with a special polymeric membrane in such a manner that only oxygen molecules are able to pass through this membrane to cause the reaction. However, this polymer membrane is extremely thin and easily damaged.

\section{Results}

Fig. 2 shows the oxygen concentration obtained immediately following intratumoral injection of PBS containing $0.5 \% \mathrm{w} / \mathrm{v}$ hydrogen peroxide. The oxygen concentration increased rapidly $\sim 4$ sec following the intratumoral injection of PBS-hydrogen peroxide.

The oxygen concentrations in the tumor tissues at $1 \mathrm{~h}$ after injection of each test agent are shown in Fig. 3. Although the oxygen concentrations in the tumor tissues had decreased compared with that of each test agent received immediately after the intratumoral injection, the oxygen concentrations were higher than the control group (PBS alone) for all agents. Furthermore, the hyaluronate- and liposome-hydrogen peroxide-injected groups were found to preserve high intratumoral oxygen pressure.

Fig. 4 shows the oxygen concentrations in the tumor tissues at $24 \mathrm{~h}$ after the injection of each test agent. In the liposomehydrogen peroxide-injected group, which had shown a high oxygen concentration $1 \mathrm{~h}$ after injection of the agent, the oxygen concentration had decreased to the level of the control group by $24 \mathrm{~h}$. The hyaluronate- and glycerol-hydrogen peroxide-injected groups were the only groups to show preserved high intratumoral oxygen pressure at $24 \mathrm{~h}$ after injection.

Thus, hyaluronate-hydrogen peroxide was identified as the most effective combination of drugs containing hydrogen peroxide to preserve high intratumoral oxygen concentration for $24 \mathrm{~h}$ following intratumoral injection of the agent.

\section{Discussion}

This study showed that intratumoral injection of hydrogen peroxide rapidly raises the oxygen concentration in the tumor tissue. Since hydrogen peroxide rapidly diffuses to the surrounding tissues, alone it results in only short-lived effects in terms of preservation of a high oxygen concentration in tumor tissue. At present, clinical radiotherapy is generally performed five times per week, and thus the development of a long-acting radiosensitizer for intratumoral injection is essential in order to decrease injection frequency. Moreover, the development of a radiosensitizer for intratumoral injection that can maintain 
a high oxygen concentration in the tumor tissue for more than $24 \mathrm{~h}$ is crucial. Its significance is due to the frequency of radiotherapy, since if the high oxygen concentration induced by the radiosensitizer is maintained from the Monday of the first radiotherapy to the Tuesday of the second radiotherapy, the frequency of injection of the radiosensitizer can be reduced to a twice-weekly basis. This reduction would be the preferred injection schedule for patients and clinicians.

At $1 \mathrm{~h}$ following intratumoral injection of each agent, the hyaluronate- and liposome-hydrogen peroxide-injected groups showed high oxygen concentrations in the tumor tissue, while at $24 \mathrm{~h}$ it was the hyaluronate- and glycerol-hydrogen peroxide-injected groups that showed high oxygen concentrations in tumor tissue. Thus, the most suitable combination of drugs for preserving a high intratumoral oxygen concentration is hyaluronate-hydrogen peroxide.

In general, sodium hyaluronate is utilized in cosmetic surgery (12) and chronic articular disorders (13), and under normal healthy conditions is found in the eyes, skin and joints. Therefore, sodium hyaluronate can be safely injected into the human body and when used as a medicine it has a pain-controlling effect. Moreover, absorption of sodium hyaluronate occurs gradually due to its strong water-holding capacity and thus its ability to maintain oxygen levels by retarding the degradation of hydrogen peroxide over a long period of time, thereby maintaining the oxygen concentration in tumor tissues.

The glycerol-hydrogen peroxide-injected group also maintained oxygen concentration in the tumor tissue at $24 \mathrm{~h}$ following injection of the agent. Glycerol is a concentrated glycerin and fructose injection liquid that is readily dissolved in water and is highly hygroscopic. Thus, glycerol-hydrogen peroxide would also be effective in maintaining tumor oxygen concentration. However, the pain-relieving effects of hyaluronate-hydrogen peroxide render it the more useful agent in the clinical setting.

In conclusion, we found that sodium hyaluronate was the most effective supporting agent for hydrogen peroxide in maintaining the oxygen concentration in tumor tissues following intratumoral injection of the hydrogen peroxide agent and for pain relief at the intratumoral injection site. Based on these results, clinical application of a novel enzyme-targeting radiosensitization treatment, known as KORTUC, has begun chiefly for malignant tumors such as advanced breast cancer, soft tissue sarcoma and cervical lymph node metastasis and has demonstrated marked therapeutic effects (14). Moreover, we have developed KORTUC III for locally advanced hepatocellular carcinoma, and KORTUC IV for locally advanced pancreatic cancer (stage IVa).

\section{References}

1. Hall EJ: The oxygen effect and reoxygenation. In: Radiobiology For The Radiologist. Hall EJ (ed). 5th edition. J.B. Lippincott Co., Philadelphia, PA, pp91-111, 2000.

2. Ogawa Y, Takahashi T, Kobayashi $\mathrm{T}$, et al: Mechanism of apoptotic resistance of human osteosarcoma cell line, HS-Os-1, against irradiation. Int J Mol Med 12: 453-458, 2003.

3. Ogawa Y, Takahashi T, Kobayashi T, et al: Apoptotic-resistance of the human osteosarcoma cell line HS-Os-1 to irradiation is converted to apoptotic-susceptibility by hydrogen peroxide: A potent role of hydrogen peroxide as a new radiosensitizer. Int $\mathrm{J}$ Mol Med 12: 845-850, 2003.

4. Ogawa Y, Takahashi T, Kobayashi T, et al: Immunocytochemical characteristics of human osteosarcoma cell line HS-Os-1: Possible implication in apoptotic resistance against irradiation. Int J Mol Med 14: 397-403, 2004.

5. Kariya S, Sawada K, Kobayashi T, et al: Combination treatment of hydrogen peroxide and $\mathrm{X}$-rays induces apoptosis in human prostate cancer PC-3 cells. Int J Radiat Oncol Biol Phys 75: 449-454, 2009.

6. Ogawa Y, Ue H, Tsuzuki K, et al: New radiosensitization treatment (KORTUC I) using hydrogen peroxide solutionsoaked gauze bolus for unresectable and superficially exposed neoplasms. Oncol Rep 19: 1389-1394, 2008.

7. Lichtenbaum R, De Souza AA and Jafar JJ: Intratumoral hydrogen peroxide injection during meningioma resection. Neurosurgery 59 (Suppl 4): 470-473, 2006.

8. Goto M, Hanyu T, Yoshio T, et al: Intra-articular injection of hyaluronate (SI-6601D) improves joint pain and synovial fluid prostaglandin E2 levels in rheumatoid arthritis: a multicenter clinical trial. Clin Exp Rheumatol 19: 377-383, 2001.

9. Yamada R, Sawada S, Uchida H, et al: Clinical study of porous gelatin sphere (YM670) in transcatheter arterial embolization. Jpn J Cancer Chemother 32: 1431-1436, 2005.

10. Teichert J, Kern HJ, Tritschler H, et al: Investigations on the pharmacokinetics of alpha-lipoic acid in healthy volunteers. Int J Clin Pharmacol Ther 36: 625-628, 1998.

11. Sakamaki M, Igarashi H, Nishiyama Y, et al: Effect of glycerol on ischemic cerebral edema assessed by magnetic resonance imaging. J Neurol Sci 209: 69-74, 2003.

12. Brandt FS and Cazzaniga A: Hyaluronic acid gel fillers in the management of facial aging. Clin Interv Aging 3: 153-159, 2008.

13. Karatosun V, Unver B, Ozden A, et al: Intra-articular hyaluronic acid compared to exercise therapy in osteoarthritis of the ankle. A prospective randomized trial with long-term follow-up. Clin Exp Rheumatol 26: 288-294, 2008.

14. Ogawa Y, Kubota K, Ue H, et al: Phase I study of a new radiosensitizer containing hydrogen peroxide and sodium hyaluronate for topical tumor injection: A new enzyme-targeting radiosensitization treatment, Kochi Oxydol-Radiation Therapy for Unresectable Carcinomas, Type II (KORTUC II). Int J Oncol 34: 609-618, 2009. 